Internist 2017 · 58:1005

DOI 10.1007/s00108-017-0273-5

Online publiziert: 9. August 2017

๑) Springer Medizin Verlag GmbH 2017

CrossMark

\author{
T. Engels \\ Tuttlingen, Deutschland
}

\title{
Vitamin-K-Antagonisten bei manifester Niereninsuffizienz kontraindiziert
}

\section{Originalbeitrag}

Rafflenbeul E, Müller-Ehmsen J (2017) Vitamin-K-Antagonisten. Ist ihre Verordnung heute wirklich ein "Kunstfehler"? Internist 58:90-99. doi:10.1007/s00108-016-0168-x

Sehr geehrter Herr Kollege Rafflenbeul, sehr geehrter Herr Professor MüllerEhmsen,

Sie formulieren im „Fazit für die Praxis“, dass es „bei schwerer Niereninsuffizienz (GFR $<15 \mathrm{ml} / \mathrm{min}$ ) bzw. Dialysepflicht ... keine orale Alternative zu VKA“ gibt.

In der Fachinformation zu Marcumar ${ }^{\circledR}$ beispielsweise wird aber eindeutig formuliert, dass der „Einsatz von VKA bei Patienten mit manifester Niereninsuffizienz kontraindiziert ist"! Hintergrund dieser Festlegung ist die unter VKATherapie beobachtete Tendenz zur Entwicklung einer Hyperkalzifikation im Gefäßsystem.

Nachdem es seit inzwischen einigen Jahren alternative Therapieoptionen in Form der neuen bzw. direkten oralen Antikoagulanzien (NOAK/DOAK) gibt, die bis zu einer glomerulären Filtrationsrate (GFR) von 30 bzw. auch $15 \mathrm{ml} / \mathrm{min}$ (Edoxaban) zugelassen sind, ist es meines Erachtens grundsätzlich nicht (mehr) vertretbar, in solchen Konstellationen eine VKA-Therapie weiter zu propagieren, und dies dann auch noch in einer derart hochrangigen $\mathrm{Pu}$ blikation. Aus meiner Sicht sollte das so formulierte „Fazit für die Praxis“

Eine Erwiderung zu diesem Leserbrief finden Sie unter doi:10.1007/s00108-017-0274-4 entsprechend korrigiert und publiziert werden!

Noch eine weitere Anmerkung: Sie heben in Ihrer Arbeit auch auf das Thema "time in therapeutic range" (TTR) ab. Für mich ist diese Problematik letztlich das im Prinzip noch eindeutigere K. o.Kriterium für den weiteren Einsatz von VKA als Alternative zu NOAK/DOAK! Wenn es denn auch mit rigoroser bzw. stringenter Kontroll- und Einstellungspraxis, wie unter anderem in Skandinavien oder den Niederlanden, kaum bis nicht wirklich gelingt, seriös akzeptable TTR-Daten- bzw. -Quoten zu realisieren, stellt sich die Grundsatzfrage, inwieweit eine derartig problematisch zu steuernde Substanz überhaupt noch eine Existenzberechtigung hat - jeder kennt die „klassischen“ Beispiele von entsprechenden Quick-/INR-Ausweisen.

Der sozioökonomische Aspekt mit der anhaltenden Tendenz zur Präferenz von VKA (!) - wie ja auch von Ihnen angesprochen - ist nach meinem Eindruck in erster Linie das Äquivalent einer ausgeprägt eigenwilligen - „kurzsichtigen“ Denkweise in den Reihen vor allem der Kostenträger! Möglicherweise wäre es pikant und reizvoll, die Experten und Entscheider im Gesundheitswesen zu fragen, ob sie im Fall des Falles für sich VKA oder dann doch lieber NOAK präferieren würden.

Wichtig scheint mir vor allem noch, aus Sicht der Internisten den operativ agierenden Kollegen notfalls brachial $\mathrm{zu}$ vermitteln, dass der immer wieder geübte Brauch des Bridging bei NOAKTherapie vor allem katastrophenträchtig ist, nachdem man diese Taktik unter gerinnungsphysiologischen Aspekten wohl mindestens als Mumpitz deklarieren muss.

Einstweilen herzliche Grüße von der Donau an die Elbe

T. Engels

\section{Korrespondenzadresse}

\section{T. Engels}

Neuhauser Str. 85, 78532 Tuttlingen,

Deutschland

engels.thomas@gmx.de

Interessenkonflikt. T. Engels gibt an, dass kein Interessenkonflikt besteht. 